\title{
Phase transitions in antiferromagnetic superlattices
}

\author{
A. S. Carriço* and R. E. Camley \\ Department of Physics, University of Colorado, Colorado Springs, Colorado 80933-7150
}

(Received 3 October 1991)

\begin{abstract}
We investigate the temperature dependence of the magnetization of films and superlattices of uniaxial antiferromagnets using a self-consistent, mean-field approach and apply the theory to $\mathrm{FeF}_{2} / \mathrm{CoF}_{2}$ superlattices. The number of magnetic phase transitions in this system (one or two) depends on the thicknesses of the films in the superlattice and on the interface exchange constant. Weak interface coupling leads to two transitions as do thick films. Our findings are in good agreement with recent experimental results and indicate that the $\mathrm{FeF}_{2} / \mathrm{CoF}_{2}$ system displays strong interface coupling.
\end{abstract}

The magnetic properties of layered structures may differ markedly from the bulk properties of the constitutive compounds. Magnetic superlattices exhibit features inherent to each of the components and new ones directly associated with the superperiodicity introduced by the alternating stacking of two materials. Also the magnetization may be strongly influenced by size effects, especially for thin-film superlattices.

In the last few years a significant amount of research has been directed to the study of metallic magnetic multilayers. This work has concentrated to a large extent on superlattices with ferromagnetic metals including $\mathrm{Fe} /$ $\mathrm{Gd},{ }^{1-4} \mathrm{Gd} / \mathrm{Y},{ }^{5} \mathrm{Fe} / \mathrm{Cr},{ }^{6-8} \mathrm{Co} / \mathrm{Ru},{ }^{8}$ and $\mathrm{Gd} / \mathrm{Dy} .{ }^{9}$ A set of results has emerged for these systems whereby a number of magnetic phases can exist and transitions between these phases can be readily introduced by small changes in temperature or applied external fields.

In contrast to the metallic systems discussed above, ionic antiferromagnetic superlattices have only very recently been constructed and studied. ${ }^{10}$ Exploring the excellent match between the lattices of nonmagnetic compounds and uniaxial antiferromagnets, a series of magnetic layered structures have been grown and characterized either by antiferromagnetic resonance ${ }^{11}$ or thermal expansion ${ }^{10}$ measurements. The extremely narrow resonance line of antiferromagnetic films has motivated a series of theoretical studies of radiation transmission under strong static magnetic fields and multistabilities in the transmission as a function of incident radiation frequency were obtained. ${ }^{12}$

In recent measurements ${ }^{10}$ on $\mathrm{FeF}_{2}(m$ layers $) / \mathrm{CoF}_{2}(n$ layers) superlattices dramatic size effects were clearly observed. The data displayed either two magnetic phase transitions or one transition depending on the composition $m / n$. Bulk $\mathrm{FeF}_{2}$ has a magnetic transition at $78.39 \mathrm{~K}$ while $\mathrm{CoF}_{2}$ has its Néel temperature at $40 \mathrm{~K}$. In thermal expansion measurements on superlattices composed of thicker films $(m / n=25 / 30)$ both the high-temperature transition for $\mathrm{FeF}_{2}$ and the low-temperature transition for $\mathrm{CoF}_{2}$ were seen. In contrast, for thinner films $(\mathrm{m} / \mathrm{n}$ $=19 / 6$ ) only a high-temperature transition is observed.

In many respects antiferromagnetic superlattices differ from the metallic ferromagnetic ones previously studied. The antiferromagnetic system tends to have a more delicate balance between exchange and Zeeman energies as a result of the cancellation of the magnetization of the two sublattices. For example, the zero-field magnetization depends strongly on the superlattice composition $m / n$ and also on the coupling of the two spin systems. In particular, odd/odd and even/even superlattices have a magnetic unit cell identical to the geometrical unit cell, and the first one exhibits a net magnetic moment at low temperatures. The even/even superlattice has no net magnetic moment as a result of the cancellation of the sublattice magnetizations in each film. In contrast, even/odd superlattices have a magnetic unit cell twice as large as the geometrical unit cell and no net magnetization at low temperatures in the absence of external fields.

In this paper we report on a theoretical study of the magnetization of films and superlattices of uniaxial antiferromagnets and make direct contact with the experimental results. We show that the number of phase transitions depends on the interface exchange constant and the thickness of the individual films in the superlattice. Our results show that when the interface exchange is strong and the $\mathrm{CoF}_{2}$ film is relatively thin, the exchange field from the $\mathrm{FeF}_{2}$ stabilizes the $\mathrm{CoF}_{2}$ magnetic structure. Thus only one magnetic phase transition is seen in this case. However, since this stabilizing effect only penetrates a few atomic layers, thicker $\mathrm{CoF}_{2}$ films are not stabilized in the center of the film and two magnetic phase transitions are seen. Our results are in good agreement with the experimental work ${ }^{10}$ and indicate that the interfacial exchange in that system must be fairly strong.

A calculation (or measurement) of magnetization does not always show the presence of the magnetic phase transitions, especially for antiferromagnets where the net magnetization may be zero. We thus calculate the magnetic contribution to the heat capacity which, similarly to the thermal expansion measurement, is sensitive to the phase transitions discussed here.

While there has been some theoretical work ${ }^{13,14}$ on phase transitions in antiferromagnetic superlattices, these were for $T=0$ and thus are not appropriate for the recent experiments. We note that there have also been some other theoretical studies on magnetization profiles in thin antiferromagnetic films. ${ }^{15}$ These works included dynamic fluctuations and are significantly more involved than the simple mean-field treatment presented here. Nonetheless, these treatments agree with our results-i.e., the magneti- 
zation is generally reduced near a free surface-excluding a small oscillation in the magnetization profile found in the dynamic treatment at low temperatures only.

The theoretical method used is similar to that described previously ${ }^{3}$ so we only sketch some of the important ideas. We use an iterative, mean-field model which allows for a variation of the magnetization profile as a function of distance from an interface or surface. In essence one begins with an arbitrary configuration giving the magnitude of the spins in each of the layers; the directions are fixed in antiferromagnetic ordering. The energy of spin, $S_{n}$, in layer $n$ is calculated as

$$
E_{n}=-g \mu_{B} S_{n} H_{n}
$$

where $H_{n}$ is the effective field produced by the anisotropy, Zeeman, and exchange energies. At finite temperature this field is given by

$$
\begin{aligned}
H_{n}= & \left(Z_{n-1} J_{n-1}\left\langle S_{n-1}\right\rangle+Z_{n+1} J_{n+1}\left\langle S_{n+1}\right\rangle\right) \\
& +H_{a}\left\langle S_{n}\right\rangle+H_{\mathrm{ext}}
\end{aligned}
$$

where $J_{n-1}$ is the exchange coupling constant connecting the $n$th layer to the $n-1$ th layer and similarly the $Z_{n-1}$ is the coordination number between spins in the $n$th and $n-1$ th layer. $\left\langle S_{n}\right\rangle$ is the thermal averaged value of the spins in the $n$th layer and is found through the use of the Brillouin function:

$$
\left\langle S_{n}\right\rangle=S B_{s}\left(E_{n} / k T\right) .
$$

The problem is, of course, to find a self-consistent set of values $\left\langle S_{n}\right\rangle$. This is done in an iterative procedure. One chooses a layer at random and finds the thermal averaged value for $\langle S\rangle$ in that layer. Another layer is then chosen at random and the thermal averaged value for that layer is also found. The process is then repeated until a selfconsistent state emerges. Generally the algorithm works quickly at low temperatures. Near the transition temperature of one of the two compounds, the number of iterations increase and appropriate expansions of the Brillouin function for small arguments are needed.

The parameters used in the calculation are as follows. For FeF $F_{2}$ the spin is $S=2$, the exchange field is $H_{\mathrm{ex}}=434$ $\mathrm{kG}$, and the anisotropy field is $H_{a}=149 \mathrm{kG}$. For $\mathrm{CoF}_{2}$ the spin is $S=1.5, H_{\mathrm{ex}}=324 \mathrm{kG}$, and $H_{a}=32 \mathrm{kG}$. These values are mean-field parameters and do not exactly match up with measured values obtained through antiferromagnetic resonance. The values used here are those which give the correct transition temperatures for $\mathrm{CoF}_{2}$ and $\mathrm{FeF}_{2}$ and which have the correct ratio between exchange and anisotropy fields. The exchange parameters, $J$, are given by the usual expression $J=g \mu_{B} H_{\mathrm{ex}} / 2 Z S$ for each material.

Thin antiferromagnetic films display clear size effects. The transition temperatures for the antiferromagnetic film may be reduced by as much as $20 \%$. Generally the bulk transition is regained with a thickness of 15 atomic layers. In Fig. 1, we show the net magnetization for $\mathrm{FeF}_{2}$ films containing 3, 5, 9, and 21 magnetic ion planes. (Since we always take an odd number of planes here, the films display a net magnetization.) The temperature axis in this figure and the following ones is scaled to the bulk

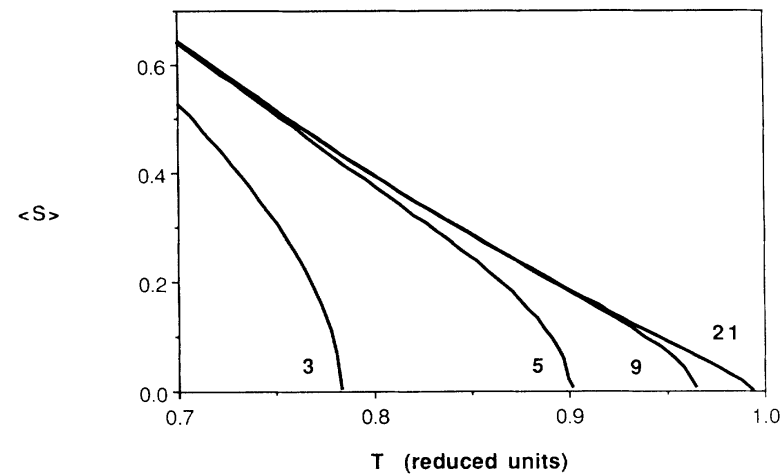

FIG. 1. Average spin value per layer for thin $\mathrm{FeF}_{2}$ films as a function of reduced temperature $T / T_{\text {Néel, }}$ where $T_{\text {Néel }}$ refers to $\mathrm{FeF}_{2}$. The numbers by the curves give the number of spin planes for each film.

Néel temperature of $\mathrm{FeF}_{2}$. It is readily seen that thin films have a reduction in the transition temperature. This reduction is also present in the superlattice case. However, the size effect is reduced by the interface coupling, especially in the strong-coupling limit.

The interaction between the two different antiferromagnetic films in the superlattice is a subject of special interest. In fact, if the compounds have very different bulk Néel temperatures, then one may, or may not, see the low-temperature transition as discussed previously. We explore this feature in Fig. 2 where we plot the calculated magnetization as a function of temperature for a $7 / 3$ $\mathrm{FeF}_{2} / \mathrm{CoF}_{2}$ superlattice with different values of interface exchange. If the interface coupling is strong, the low Néel temperature compound can be stabilized by the other component of the superlattice, resulting in a much slower decay of the magnetization with temperature. In this case the net magnetization decreases smoothly with temperature and only the high-temperature transition is seen. In contrast, if the interface coupling is weak, the spin systems are effectively independent and two transitions are seen. The low-temperature transition is seen as a rapid increase in the net magnetization for this structure.

It is apparent from the results above that certain phase

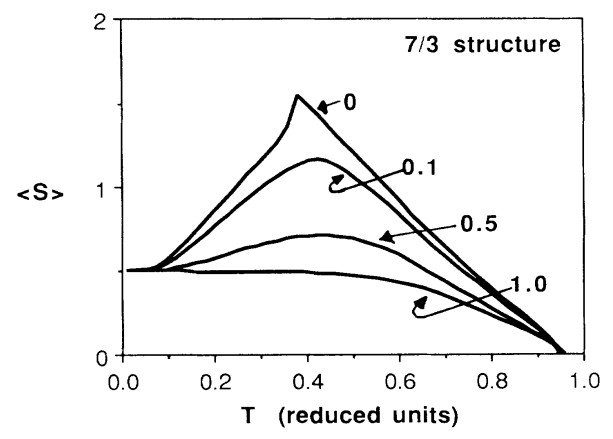

FIG. 2. Thermal averaged net spin values per unit cell for $\mathrm{FeF}_{2} / \mathrm{CoF}_{2}$ superlattice for a $7 / 3$ structure and as a function of temperature for different interface exchange coupling. The numbers give the ratio $J_{\text {interface }} / J_{\mathrm{FeF}_{2}}$. 

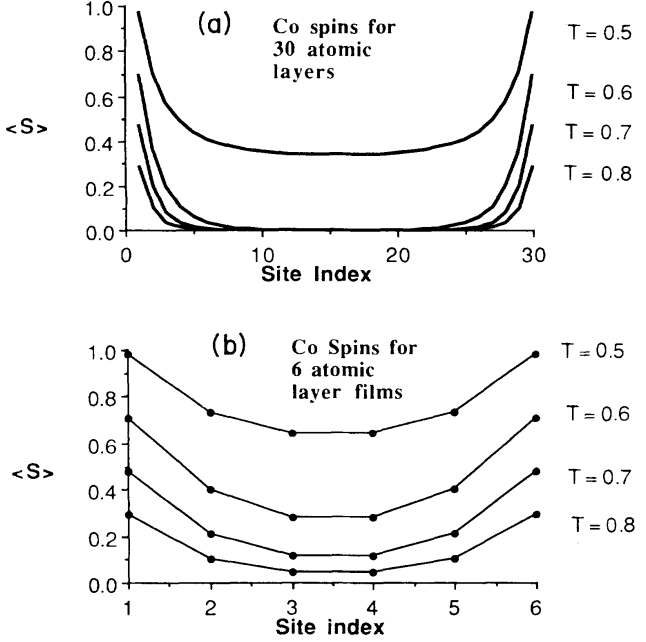

FIG. 3. Absolute value of the $\mathrm{CoF}_{2}$ spins as a function of position for the $\mathrm{FeF}_{2} / \mathrm{CoF}_{2}$ superlattice with structures of $25 / 30$ and 19/6. We assume strong interface coupling. Note that the structure with only 6 layers of spins remains in ordered state over the entire temperature range.

transitions can be clearly seen in the temperature dependence of the superlattice magnetization. However there is an interesting possibility that does not fulfill this criteria. This is closely related to the antiferromagnetic character of the system and consists of a phase transition involving a set of spins with no net magnetization. For thick $\mathrm{CoF}_{2}$ films, it is possible for spins in the central part of the $\mathrm{CoF}_{2}$ film to undergo a phase transition at about the $\mathrm{CoF}_{2}$ bulk Néel temperature regardless of the value of the interface exchange.

In Fig. 3 we show the $\mathrm{CoF}_{2}$ spin profiles (magnitude as a function of position) for the 19/6 and 25/30 structures for four selected temperatures. These values are chosen in order to make a direct comparison with the recent experiments. The interfacial exchange was taken to be the $\mathrm{FeF}_{2}$ value. This represents the strong-coupling limit and effectively means the $\mathrm{CoF}_{2}$ interface spins are subjected to an exchange field twice as large as the bulk $\mathrm{CoF}_{2}$ exchange. It is readily seen from Fig. 3 that the two structures behave quite differently. The six $\mathrm{CoF}_{2}$ spins remain nonzero over the whole temperature range, whereas a major portion of the $25 \mathrm{CoF}_{2}$ spins clearly drop to zero at about $T=0.6(46 \mathrm{~K})$. Thus the siructure with the thinner $\mathrm{CoF}_{2}$ film remains in an ordered state well beyond the bulk transition temperature of $\mathrm{CoF}_{2}$. It is clearly seen that in this case the $\mathrm{CoF}_{2}$ spins near the interface are stabilized by the exchange coupling to the $\mathrm{FeF}_{2}$.

The transition discussed above will not be seen in either a calculation or a measurement of the net magnetization of the superlattice. However, it is readily seen in the thermal expansion measurements. We point out that it can also be directly seen by examining the magnetic contribution to the heat capacity. The heat capacity can be easily calculated from our theoretical model once the equilibrium structure is known. One simply evaluates the

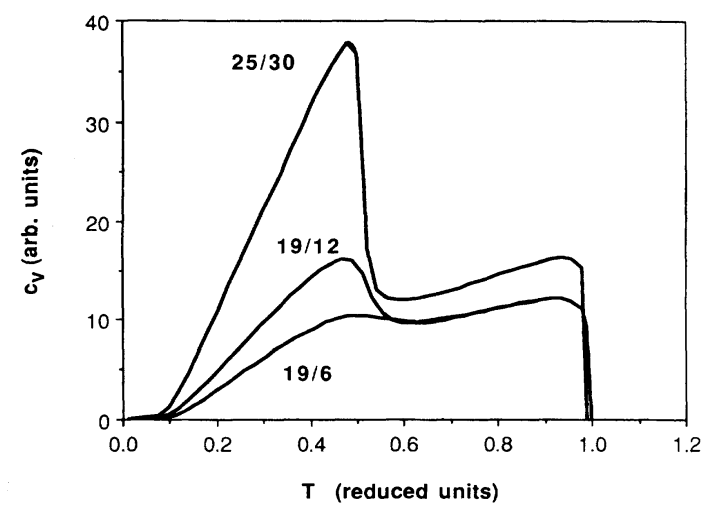

FIG. 4. Magnetic contribution to the heat capacity as a function of reduced temperature. For the structures with thicker $\mathrm{CoF}_{2}$ films, two phase transitions are seen, while for the structures with the thinner $\mathrm{CoF}_{2}$ films, only one transition is seen.

average energy at a given temperature, and then numerically takes the derivative $c_{v}=d U / d T$ by taking the difference of the energies at nearby temperatures. The magnetic heat capacity for a variety of structures in the strong-coupling limit is shown in Fig. 4. For the structures which have a phase transition (19/12 and 25/30) we see two distinct peaks, each followed by a very rapid decrease in the heat capacity. The sharp decreases are clear manifestations of the high- and low-temperature phase transitions. In contrast, the structure that does not have a phase transition shows a very different behavior, a small rounded shoulder, in the low temperature region where the phase transition might occur.

While a calculation of the thermal expansion coefficient as a function of temperature is beyond the scope of this paper, we note that the behavior of the thermal expansion coefficient is generally similar to that of magnetic heat capacity. ${ }^{16}$ The heat capacity curves calculated here do show the same general features seen in the thermal expansion coefficient curves found experimentally, particularly a clear peak for the $\mathrm{CoF}_{2}$ transition for the superlattices with thicker films and only a rounded shoulder for the superlattices with thinner films.

In summary, the observed features of the $\mathrm{FeF}_{2} / \mathrm{CoF}_{2}$ superlattices can be readily understood in terms of a simple model. For thin $\mathrm{CoF}_{2}$ films strong interface exchange coupling can stabilize a few Co spins near the interface and prevent a phase transition. For thicker $\mathrm{CoF}_{2}$ films a bulk-type phase transition takes place in the center of the film, independently of the value of the interface exchange. We point out that although these phase transitions cannot be seen by a direct measurement of the magnetization, they can be observed in a measurement of the heat capacity.

The work of A.S.C. was financed by the Fulbright Commission, the Brazilian Research Council (CNPq), and the ARO. The work of R.E.C. was supported by the U.S. Army Research Office under Grant No. DAALO3-91-G-0299. 
${ }^{*}$ On leave from Departamento de Fisica, Universidade Federal do Rio Grande do Norte, 59072 Natal/Rn Brazil.

'Experimental work connecting magnetization and magnetoresistance in this structure can be found in $\mathrm{H}$. Fujimori, Y. Kamiguchi, and Y. Hayakawa, J. Appl. Phys. 67, 5716 (1990); Y. Kamiguchi, Y. Hayakawa, and H. Fujimori, Appl. Phys. Lett. 55, 1918 (1989).

${ }^{2}$ S. Tsunashima, T. Ichikawa, M. Nawate, and S. Uchiyama, J. Phys. (Paris) Colloq. (Suppl.) 49, C8-1803 (1988).

${ }^{3}$ R. E. Camley and D. R. Tilley, Phys. Rev. B 37, 3413 (1988); R. E. Camley, ibid. 39, 12316 (1989).

${ }^{4} \mathrm{~A}$ careful study of the magnetic phase diagram in $\mathrm{Fe} / \mathrm{Gd}$ has been carried out by K. Cherif, C. Dufour, Ph. Baur, G. Marchal, and Ph. Mangin, Phys. Rev. B 44, 7733 (1991).

${ }^{5}$ J. Kwo, M. Hong, F. J. DiSalvo, J. V. Waszczak, and C. F. Majkrzak, Phys. Rev. B 35, 7925 (1987).

6P. Grünberg, R. Schreiber, Y. Pang, M. B. Brodsky, and H. Sowers, Phys. Rev. Lett. 57, 2442 (1986).

${ }^{7}$ J. J. Krebs, P. Lubitz, A. Chaiken, and G. A. Prinz, Phys. Rev. Lett. 63, 1645 (1989).
${ }^{8}$ S. S. P. Parkin, N. More, and K. P. Roche, Phys. Rev. Lett. 64, 2304 (1990).

${ }^{9}$ R. E. Camley, J. Kwo, M. Hong, and C. L. Chien, Phys. Rev. Lett. 64, 2703 (1990).

${ }^{10}$ C. A. Ramos, D. Lederman, A. R. King, and V. Jaccarino, Phys. Rev. Lett. 65, 2913 (1990).

I'M. Lui, J. Drucker, A. R. King, J. P. Kotthaus, P. K. Hansma, and V. Jaccarino, Phys. Rev. B 33, 7720 (1986); C.A. Ramos, A. R. King, and V. Jaccarino, ibid. 40, 7124 (1989).

${ }^{12}$ N. S. Almeida and D. L. Mills, Phys. Rev. B 36, (1987); N. S. Almeida and A. S. Carriço, Solid State Commun. 72, 181 (1989), and references therein.

${ }^{13}$ L. L. Hinchey and D. L. Mills, J. Appl. Phys. 57, 3687 (1985).

${ }^{14}$ L. L. Hinchey and D. L. Mills, Phys. Rev. B 33, 3329 (1986); 34, 1689 (1986).

${ }^{15}$ H. T. Diep, Phys. Rev. B 43, 8509 (1991). This work also contains references to other dynamic treatments of antiferromagnetic films and superlattices.

${ }^{16}$ S. Chikazumi and S. Charap, Physics of Magnetism (Krieger, New York, 1978), p. 178. 